\title{
Thermodynamics-Structure-Dynamics Correlations and Nonuniversal Effects in the \\ Elastically Collective Activated Hopping Theory of Glass-Forming Liquids
}

\author{
Baicheng Mei ${ }^{1,4}$, Yuxing Zhou ${ }^{1,4}$ and Kenneth S. Schweizer ${ }^{1-4, *}$
}

Department of Materials Science ${ }^{1}$, Department of Chemistry ${ }^{2}$ and Department of Chemical \&

Biomolecular Engineering ${ }^{3}$, Materials Research Lab ${ }^{4}$, University of Illinois, Urbana, IL 61801

*kschweiz@illinois.edu

Additional results relevant to discussions in the main text.

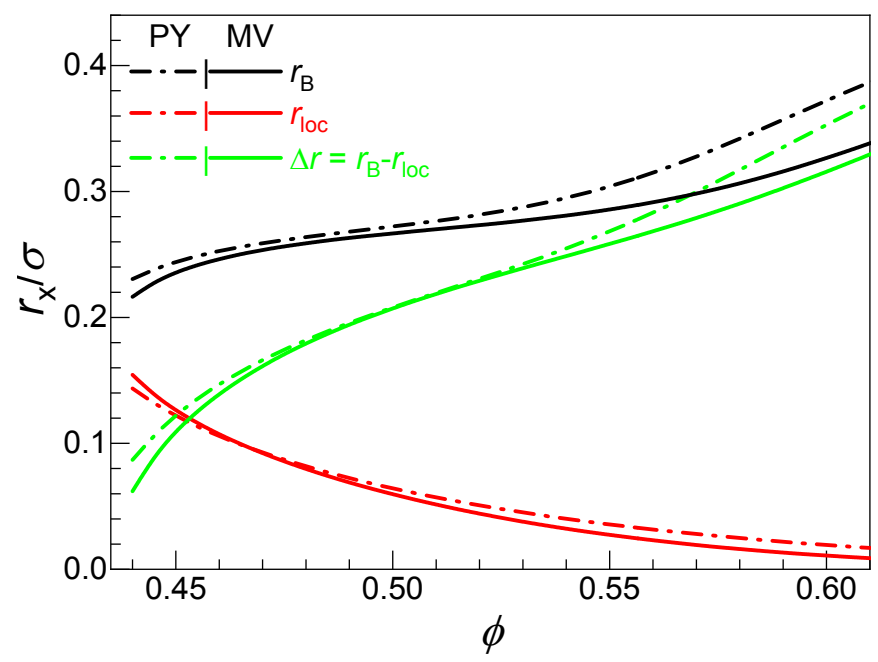

Figure S1. Localization length (red), barrier location (black), and particle jump distance (green) in units of the hard sphere diameter as a function of packing fraction based on PY (dash-dot) and MV (solid) structural inputs. 


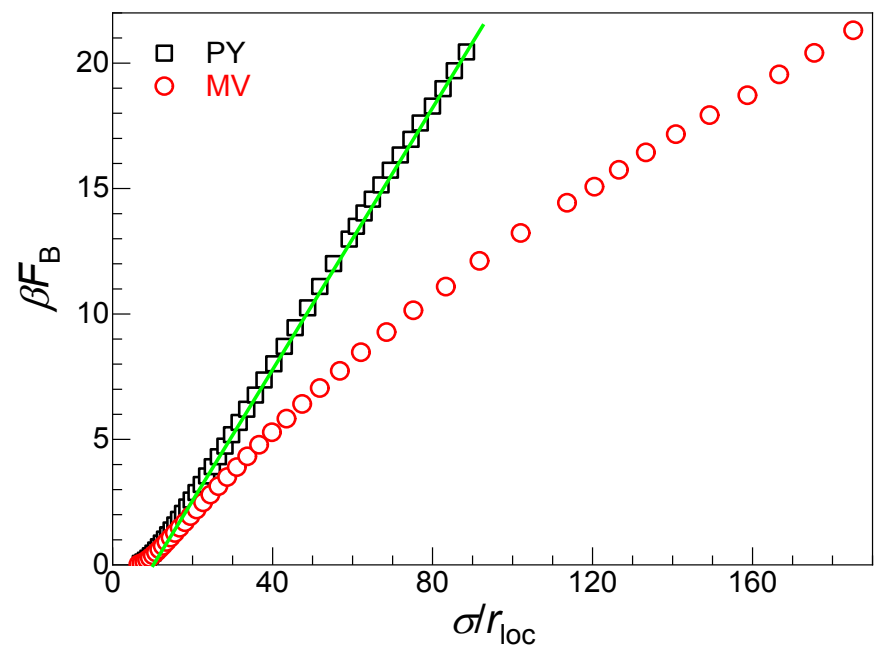

Figure S2. Local cage barrier in thermal energy units versus dimensionless inverse dynamic localization length based on PY (black) and MV (red) inputs.

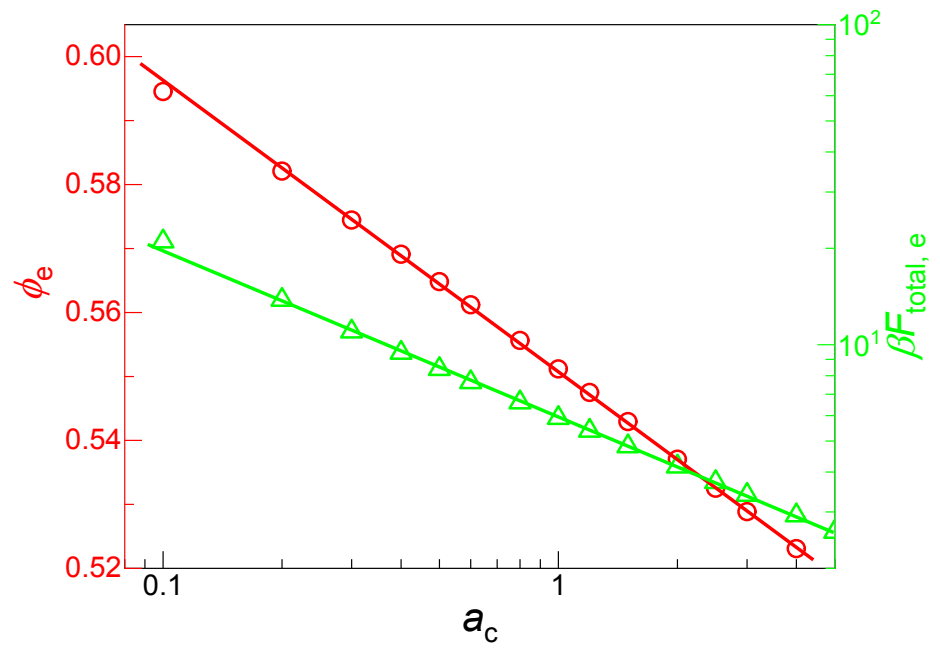

Figure S3. Dependence of the packing fraction $\phi_{e}$ (red) and corresponding total barrier $\beta F_{\text {total }, e}$ (green) at the dynamic crossover when the collective elastic barrier equals $1 k_{\mathrm{B}} T$. The straight lines are exponential (red) and power law (green) fits given by : $a_{c} \sim \exp \left(-50.53 \phi_{e}\right)$ and $\beta F_{\text {total }, e} \sim a_{c}^{-0.52}$ 


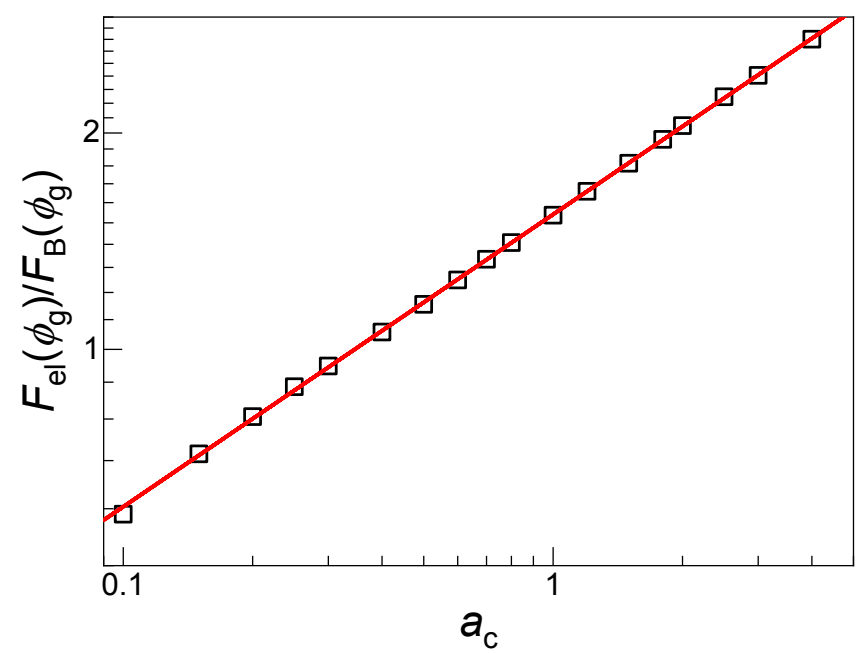

Figure S4. Ratio of the collective elastic to local cage barriers at kinetic vitrification (total barrier of 32) as a function of $a_{\mathrm{c}}$ based on MV structural input. The straight line corresponds to the power law fit: $F_{\mathrm{el}}\left(\phi_{\mathrm{g}}\right) / F_{\mathrm{B}}\left(\phi_{\mathrm{g}}\right)=1.54 a_{\mathrm{c}}^{0.41}$. 